\title{
Rigging the deck: Selecting good problems for expert-novice card-sorting experiments
}

\author{
Steven F. Wolf, ${ }^{1,2,3, *}$ Daniel P. Dougherty, ${ }^{2, \dagger}$ and Gerd Kortemeyer ${ }^{1,2, \ddagger}$ \\ ${ }^{1}$ Department of Physics and Astronomy, Michigan State University, East Lansing, Michigan 48824, USA \\ ${ }^{2}$ Lyman Briggs College, Michigan State University, East Lansing, Michigan 48825, USA \\ ${ }^{3}$ CREATE for STEM Institute, Michigan State University, East Lansing, Michigan 48824, USA
}

(Received 6 July 2012; published 12 November 2012)

\begin{abstract}
A seminal study by Chi et al. firmly established the paradigm that novices categorize physics problems by "surface features" (e.g., "incline," "pendulum," "projectile motion," etc.), while experts use "deep structure" (e.g., "energy conservation," "Newton 2," etc.). Yet, efforts to replicate the study frequently fail, since the ability to distinguish experts from novices turns out to be highly sensitive to the problem set being used. Exactly what properties of problems are most important in problem sets that discriminate experts from novices in a measurable way? To answer this question, we studied the categorizations by known physics experts and novices using a large, diverse set of problems. This set needed to be large so that we could determine how well experts and novices could be discriminated by considering both small subsets using an exhaustive Monte Carlo approach and larger subsets using simulated annealing. We found that the number of questions required to accurately classify experts and novices can be surprisingly small so long as the problem set is carefully crafted to be composed of problems with particular pedagogical and contextual features. Finally, we found that not only was what you ask (deep structure) important, but also how you ask it (problem context).
\end{abstract}

DOI: 10.1103/PhysRevSTPER.8.020116

\section{INTRODUCTION}

The physics education research (PER) community has been grappling with different understandings of student learning and the conceptualization of expertise. The predominant paradigm that the PER community has used to understand expertise was established by the seminal study done by Chi et al. [1]. However, replicating this experiment has been challenging, and, as an informal survey among physics education researchers has indicated, fails more often than not. To understand this phenomenon, we have designed and carried out a categorization experiment and developed a novel methodology to analyze that experiment [2]. We describe each sorter's categorization as a graph, quantify the difference between any two categorization graphs using our distance metric, and visualize the relative position of each of the sorters using principal component analysis (PCA) [2].

We found that the most salient feature of problem categorizations, that is, the first principal component of the PCA, is related to the sorting behavior of the individuals, a personality trait we termed "stacker" versus "spreader" [2]. The meaning of this component was easy

\footnotetext{
*wolfste4@msu.edu

†oughe57@msu.edu

*korte@lite.msu.edu
}

Published by the American Physical Society under the terms of the Creative Commons Attribution 3.0 License. Further distribution of this work must maintain attribution to the author(s) and the published article's title, journal citation, and DOI.
PACS numbers: 01.40.Fk, 01.40.Ha, 01.55.+b, 01.90.+g

to recognize, as it exhibited itself in the stark visual differences between the sorters' categorization graphs. A stacker tended to generate a few large piles of cards with the same problem being a member of several piles. In contrast, a spreader tended to generate many fine-grained small piles and would rarely allow a single problem to be a member of several piles. Expertise only came in second, as the distinction between experts and novices is exhibited by the second principal component. Unfortunately, due to the multidimensional nature of the principal component analysis and missing visual clues in the graphs, we were unable to infer the source of this variability. However, all indications are that this behavior is problem specific. After all, variability in student performance while working on problems in relativity [3] and motion [4] is well documented. It is reasonable to believe that this problem-dependent nature of student reasoning extends from solving problems to the categorization behavior of sorters, as it depends on the particular problems being used. What is the composition of a minimal ideal subset? What problem features need to be present? In other words, instead of picking random problems from the back of chapters in textbooks, how much does a problem set need to be "rigged" in order to be effective in discriminating experts and novices?

Additional evidence for the importance of careful question selection-or "rigging" — was given by Veldhuis [5], who also attempted to verify the result of Chi et al. Veldhuis created four different categorization sets. The first set was created in an attempt to mimic the Chi et al. problem set [6], and the second was a control set with a 
similar collection of end-of-chapter problems. In contrast, the third and fourth sets were carefully constructed so that each problem had only a single physics principle and a single surface feature from a set of four principles and four surface features [5]. The fourth set was also "rigged." It had the same number and type of cards, but only two surface and two conceptual features. Veldhuis could not draw a conclusion from the categorizations from his first two problem sets. However, sets 3 and 4 agreed with Chi et al. in that experts categorize problems based on physics principles while novices show a "more complex behavior" $[5,6]$. Where on the "rigging continuum" do problem sets need to be constructed in order to achieve measurable results? Also, can Veldhuis's result be generalized to more complex problems-that is, problems with more than one physics principle and more than one surface feature?

We explore on a microscopic level why particular sets of problems would discriminate experts from novices while others do not, looking at the individual properties of the included problems. The main strategy of our approach is to pick subsets out of our large set of problems, determine how well they distinguish experts from novices, and then examine their composition based on a number of pedagogical and contextual features. Combinatorics dictate that we can effectively sample the entire population for small subsets only, while for larger subsets, we use simulated annealing to optimize selected "starter sets." We do not propose that subset analysis is equivalent to actually giving our sorters many subsets to categorize. Yet, we believe that subset analysis is still able to find the features that should be present in an ideally "rigged" problem set.

\section{EXPERIMENTAL PARAMETERS}

Previously, we designed and carried out a card-sorting experiment on physics experts and novices at Michigan State University (MSU), adhering to the experimental method of Chi et al. as closely as possible [2]. A total of 18 physics professors and 23 novices participated in our study. All of the novices had completed at least the first semester of an introductory physics course at MSU. We gave each sorter a set of 50 problems to sort based on "similarity of solution," explicitly following the prompt of Singh [7]. Each sorter categorized his or her problems and recorded the groups and group names in a separate packet. Multiple categorization, i.e., putting a single card into more than one category, was allowed, but not expected.

Our previous study was able to differentiate experts from novices using this categorization task [2]. However, we were not able to explain the source of the variation between these two groups. The reason for this was due to our implementation of the PCA algorithm. In general, PCA analyzes multidimensional data and rotates the coordinate system in such a way that most of the variability is contained in the first few (often two or three) dimensions. These optimized dimensions are called the principal components and are numbered so that the first principal component (PC1) is the dimension which explains the most variability in our data set, the second principal component (PC2) explains the next most variability in our data set, and (a)

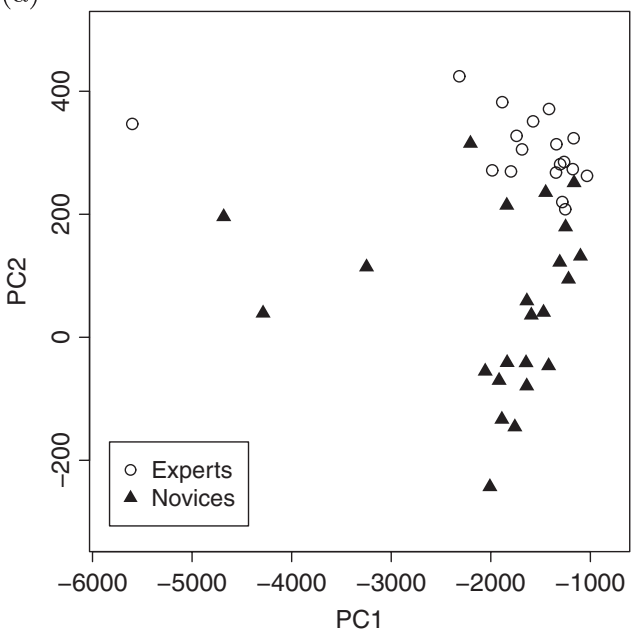

(b)

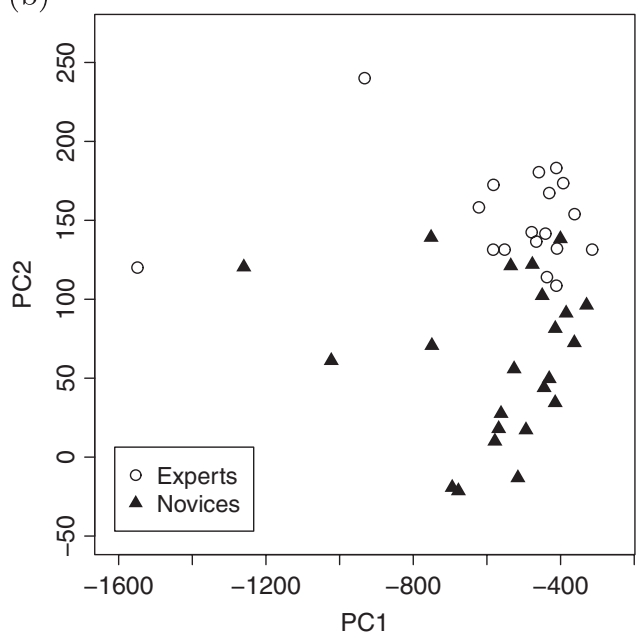

FIG. 1. (a) The PCA plot of the sorters for the entire set of problems from our previous study [2]. Both a Cramer's test ( $p=0.048$ ) and a Hotelling's test $\left(p<10^{-5}\right)$ find the expert and novice groups to be distinct at a 95\% confidence level. (b) The PCA plot of the sorters considering only the problems from Singh's study. Both a Cramer's test $(p=0.041)$ and a Hotelling's test $\left(p<10^{-5}\right)$ find the expert and novice groups to be distinct at a 95\% confidence level. For both plots, PC1 is the coordinate along the first principal axis, and PC2 is the coordinate along the second principal axis. Sorters known by us to be experts are marked by circles while sorters known by us to be novices are marked by filled triangles. The second principal component discriminates experts from novices, as experts congregate higher on this axis than novices. 
this is carried out for as many dimensions as the researcher wishes to visualize.

Oftentimes, PCA analyzes multivariable data in a matrix which is often formatted in such a way that each row is an observation and each column is a statistical property. For example, someone wishing to compare the states in the U.S. would use different economic and demographic statistics for each state. Each row would be the data for a specific state (e.g., Michigan) and each column would be a specific statistic (e.g., total population). With this sort of data, the PCA is immediately interpretable. That is, one can analyze each principal component (e.g., PC1 is $40 \%$ due to population density, $30 \%$ due to median household income, $20 \%$ due to minority population fraction, and $10 \%$ due to other factors). In our application this is not possible due to the fact that we are analyzing a distance matrix. The $j$ th column is a measurement of "the distance from sorter $j$." While we are limited from using PCA as an interpretive tool, we may still use it as a visualization tool. [See Fig. 1(a).]

At this point the results of our previous study are both tantalizing and deeply unsatisfying. After all, what is the point of being able to discriminate expert from novice without understanding the differences between these groups? In order to interpret the discrimination found by the PCA, we have studied subsets of our large initial set of problems. We have focused on two groups of statistics for each of the subsets chosen: The pedagogical and contextual features of the problems within each subset, as well as the ability of each subset to discriminate experts from novices using our analysis method. These two statistical groups will be discussed in the subsequent sections.

\section{A. Problem set creation}

We constructed our initial large set of problems such that it was diverse in terms of both content and cognitive demands. We considered two traditional measures for describing each problem. First, we used the chapter which

TABLE I. Chapter titles taken from Walker's textbook as a representative list.

\begin{tabular}{ll}
\hline \hline Chapter no. & Chapter title \\
\hline 1 & Introduction \\
2 & One-Dimensional Kinematics \\
3 & Vectors in Physics \\
4 & Two-Dimensional Kinematics \\
5 & Newton's Laws of Motion \\
6 & Applications of Newton's Laws \\
7 & Work and Kinetic Energy \\
8 & Potential Energy and Conservative Forces \\
9 & Linear Momentum and Collisions \\
10 & Rotational Kinematics and Energy \\
11 & Rotational Dynamics and Static Equilibrium \\
\hline \hline
\end{tabular}

TABLE II. A limited hierarchy of the cognitive processes described by the TIPP. Most problems in a standard physics textbook require comprehension-integrating as the highest cognitive process required involving declarative knowledge and retrieval-executing as the highest cognitive process required involving procedural knowledge [9]. The level indicates the numeric value we scored the highest cognitive process required by each problem.

\begin{tabular}{llc}
\hline \hline Cognitive process & Subprocess & Level \\
\hline Retrieval & Recall or recognize & 1 \\
& Executing $^{\text {a }}$ & 2 \\
Comprehension & Integrating & 3 \\
& Symbolizing & 4 \\
Analysis & Matching & 5 \\
& Classifying & 6 \\
& Analyzing errors & 7 \\
\hline \hline
\end{tabular}

${ }^{\mathrm{a} P r o c e d u r a l ~ k n o w l e d g e ~ o n l y . ~}$

a problem is in (using Walker's textbook [8] as a guide). See Table I for a list of chapter titles. Second, we used the problem difficulty as measured by the number of "dots" a problem would have in the back of a standard physics textbook. We also included the taxonomic classification according to the Taxonomy of Introductory Physics Problems (TIPP) [9]. The TIPP is useful because it considers two dimensions or knowledge domains, one for declarative knowledge (information) and the other for procedural knowledge (mental procedures) [9]. See Table II for a list of the TIPP levels included in our study. Higher levels were not included because they are more suited to research projects rather than homework problems [9]. Each problem was therefore classified along four feature dimensions: the chapter in which the problem could be found (CHAP), the problem difficulty (DIFF), as well as the highest complex cognitive process necessary to solve it for both declarative knowledge (TIPP-D) and procedural knowledge (TIPP-P). We did not consider the surface features (as used by Veldhuis [5]) of the problems, since those are not quantifiable.

\section{B. Expert-novice differentiation}

Our initial experiment found that experts and novices were separated by the second principal component. While this is visually possible based on figures like Fig. 1, where the experts congregate higher on the PC2 axis than the novices, we needed a way to quantify this differentiation. We considered three statistical tests: the Hotelling test [10], the Cramer test [11], and the average rate of correct classification (ARCC) [12]. Hotelling's $T^{2}$ statistic is a multivariate generalization of the well-known student's $T$ statistic. Just as the well-known two-sample $T$ statistic adjusts for degrees of freedom and variance in the data being compared, the confidence levels predicted by the 
Hotelling $T$-squared statistic are adjusted in relation to the degrees of freedom and the uncertainty (by way of multivariate covariance) in the data being compared. The Cramer test is a nonparametric analog of the Hotelling test; that is, it does not assume a distribution shape [11]. Importantly for our application, the Cramer test can be more statistically powerful than Hotelling's $T^{2}$ test when the standard distributional assumptions of the $T$-squared statistic are not met (e.g., independence of samples, multivariate normality, equal variances or covariances). The ARCC is a statistical test which relies on linear discriminant analysis to determine how well experts and novices are separated by the PCA.

We combined all of these measures into a canonical correlation analysis (CCA) [13]. A CCA quantifies the relationship between the predictor variables (in our case the problem statistics) and the explanatory variables (the sorter discrimination statistics). Because CCA assumes linear relationships between the predictor variables and the response variables, we did a log transformation on all of our variables in order to linearize power-law relationships and symmetrize skewed distributions. When analyzing the results of the CCA, we found that the Cramer statistic was an order of magnitude more important than the Hotelling and ARCC statistics. Thus, we ended up using just the Cramer statistics as the CCA for this study. In order to determine which problem properties are most important for predicting sorter discrimination, we found the variability explained by each problem feature dimension (CHAP, DIFF, TIPP-D, and TIPP-P) in this manner:

$$
\operatorname{var}_{\text {stat }}=\frac{\text { cor }_{\text {stat }}}{\sum_{\text {stats }} \operatorname{cor}_{\text {stat }}}
$$

where cor $_{\text {stat }}$ is the correlation coefficient found by calculating a CCA for each group of problem statistics with the sorter discrimination statistics and the summation in the denominator is done over the groups of statistics.

\section{SUBSET ANALYSIS}

Can subsets be as effective as the complete set of 50 cards? As a proof of concept, we have compared the sorter visualizations from the entire data set from our previous study [2] to the subset of problems within that set which we obtained from Singh's study [7]. As can be seen in Figs. 1(a) and 1(b), the two visualizations have similar properties. Naturally, the sorters have different relative positions, as our distance metric, and consequently our visualization method, was designed to compare categorizations on a microscopic-or problem-specific-level. However, the overall level of expert-novice discrimination is similar, so the smaller carefully chosen subset would have been sufficient to discriminate experts from novices.

\section{A. Monte Carlo analysis}

When forming subsets of our 50-problem set, combinatorics limit the sizes of subsets for which we can explore possible combinations. We analyzed 40000 five-problem subsets and 275000 ten-problem subsets of our original 50 -problem set. These numbers of subsets were chosen so that we could be $99 \%$ sure we had at least one five-problem subset with 5 of the 10 best problems in it and one tenproblem subset with 10 of the best 20 problems. This choice has allowed us to effectively sample the populations of five-problem and ten-problem subsets As these were random subsets, they were quite diverse in terms of the features of the problems within each subset.

In our analysis of the subsets of problems, we found that the ability of these subsets to distinguish experts from novices varied from negligible levels to nearly total separation. Moreover, this behavior was prevalent for both the five-problem and ten-problem subsets. Using a CCA, we quantified the relationship between the problem statistics and the sorter discrimination statistics for the five-problem and ten-problem subsets independently. For the fiveproblem subsets, we found a correlation coefficient of $r^{2}=$ 0.359 , while for the ten-problem subsets, we found $r^{2}=0.427$. This means that the CCA can account for $35.9 \%$ of the variability in the five-problem subset analysis and $42.7 \%$ of the variability in the ten-problem subset analysis. Given this result, it is clear that there was a problem set size effect on the ability to discriminate experts from novices. However, caution is warranted as discussed in the Introduction as subset analysis is not the same as repeating the experiment with fewer cards.

Investigating the relationships found by the CCA further, we found that the most variability is contained in the chapter variables, followed by the TIPP-P variables (see Table III). The fact that chapter variables explained a great deal of variability was not surprising since this was our analog for "deep structure" (i.e., there is a force chapter, an energy chapter, a momentum chapter, etc.). However, more interesting was the prominence of TIPP-P in explaining expert-novice sorting differences. Therefore, it was important that the problem set under consideration ask questions that require more than calculation and include tasks such as making a flow chart of a problem solving strategy. It is possible that the prominence of the TIPP-P

TABLE III. Percentage of the known variability explained by each of our problem variable groups among our ten-problem subsets. From this we see that the chapter was an important variable, followed by the TIPP-P statistic.

\begin{tabular}{lc}
\hline Problem variable group & Percent variability explained \\
\hline Chapter & 41.4 \\
TIPP-P & 30.4 \\
Difficulty & 22.8 \\
TIPP-D & 5.4 \\
\hline \hline
\end{tabular}


statistic is due in part to the nature of the students at MSU. As the physics courses are in a large lecture format and require computerized homework, questions that require hand-grading are few. Therefore, these sorts of problems may have "surprised" our novice sorters, which may have affected their ability to sort these problems. Problem difficulty was the next most important statistic. Here we found that the "easy" problems, as determined by a typical textbook author, were the most important in discriminating expert from novice. However, it is also possible that these problems were deceptively easy, or that the novices were overthinking the problem. Table III also clearly indicates that the TIPP-D level does not play a large role in discriminating experts from novices.

\section{B. Simulated annealing analysis}

As described earlier, the number of subsets to study grows quickly as the subset size grows (up to 25-problem subsets), and the sheer size of this search space limited exhaustive algorithms to ten-problem subsets. However, most categorization studies have looked at more problems $[1,5,7,14]$, and we needed to find an optimization algorithm to analyze larger sets. To minimize the problem of being "trapped" in local minima which can trap typical optimization algorithms, we are using simulated annealing $[15,16]$. Simulated annealing's optimization routine is based in principle on the metallurgical annealing process whereby impurities are removed from a metal by heating it. If you assume that the parameter that you are interested in minimizing corresponds to the "energy," we allow the algorithm to move to a new state with a probability

$P\left(E_{\text {old }}, E_{\text {new }}, T\right)= \begin{cases}1 & \text { when } E_{\text {new }}<E_{\text {old }} \\ \exp \left(\frac{E_{\text {old }}-E_{\text {new }}}{T}\right) & \text { elsewhere, }\end{cases}$

where $T$ is a "temperature" which is decreasing with every iteration of the code. For each iteration, we generated new problem sets to study by replacing two problems at a time. Each run consisted of 30000 iterations. As the Cramer statistic and its critical value depends on the distribution of the groups that are being compared, in order to make a uniform comparison, we needed to scale the Cramer statistic by its critical value. This critical value gives the minimum value for the Cramer test to distinguish the two groups. The energy $E$ that we minimized was the reciprocal of the Cramer statistic ratio, specifically,

$$
E=\frac{\text { critical value of Cramer statistic }}{\text { Cramer statistic }}
$$

Since no Cramer Statistic was more than 10 times its critical value, this measure did not have the difficulty of being too close to zero.
As the problem set used by Singh was included in our set, we have used that as a starting point for the simulated annealing algorithm, as well as 50 randomly chosen subsets of the same size ( 25 problems). To extrapolate the results from the ten-problem subsets to 25-problem subsets, we compared the problem statistics and the sorter discrimination statistics for each of these groups of subsets. Since (except for the Singh subset), the ten-problem and 25-problem subsets were chosen at random, we expected that the groups would initially not have significantly different properties. Indeed, we found that these groups are not statistically distinguishable in terms of problem features $(p=0.5621)$ or sorter discrimination statistics $(p=0.2951)$.

How much optimization could we achieve by simulated annealing? The problem feature coordinates of the optimal subsets were measurably larger $\left(p<10^{-8}\right)$ than the initial random subsets (the Singh subset and its "optimized" version did not follow this trend). Not surprisingly, as the algorithm looked for maximum sorter discrimination, we found that the optimal sets had measurably larger $\left(p<10^{-15}\right)$ sorter discrimination statistics than the random subsets as well.

As the optimal problem sets found with different starting subsets are not identical, we cannot say that we have found a global optimum, only many local optima instead. However, based on the results from the exhaustive analysis of the ten-problem subsets, the optimized 25-problem subsets are in the top $2 \%$ of all possible sorter discrimination statistics.

How much "rigging" happened as the result of optimization; i.e., how different are optimized sets from random ones? If we indicate the set of problems that we started with as $S$, and the optimized (or best) set of problems to be $B$, we define the rigging fraction to be

$$
R=\frac{\text { length }(S \cap B)}{\text { length }(S)},
$$

where the length simply gives the number of elements in the set, and the rigging fraction $(R)$ is the fraction of initial problems retained in the optimized set. The rigging fraction is therefore a comparison between a starting set of problems and the nearest local optimum for that set of problems.

To get a feel for the quality of this measure, we again used it first on the Singh subset. Singh noted that she chose her problems carefully: "Many questions ... were chosen ... because the development of these questions and their wording had gone through rigorous testing by students and faculty members" [7]. Indeed, the rigging fraction of the Singh subset was larger than the rigging fraction of any of the random subsets (see Fig. 2), which assured us that we found a good measure.

Thus, we found that regular-size 25-problem subsets also require "rigging," and that they should be optimized 


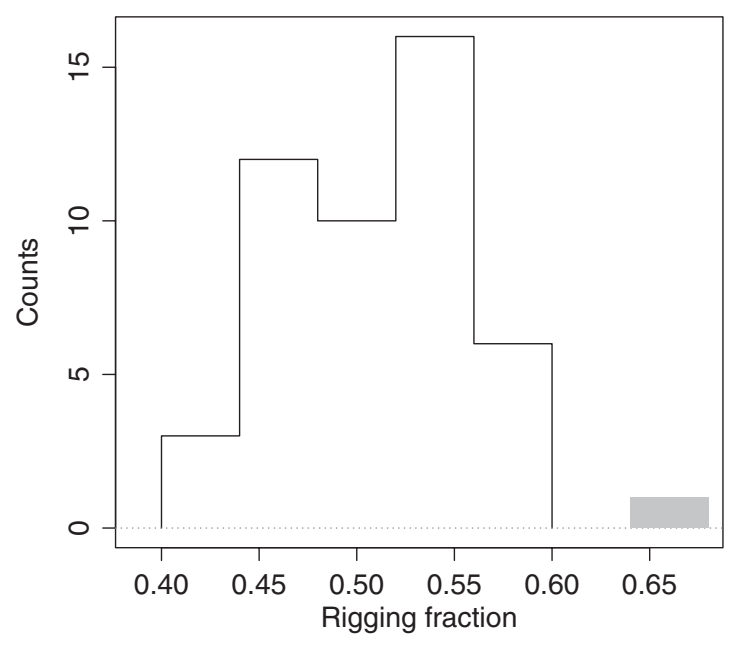

FIG. 2. Histogram of the rigging fraction for the subsets with a random starting point. The shaded gray box indicates the rigging fraction of the Singh subset. From this, we can see that the Singh subset has more problems in common with its nearest local optimum than do any of the random subsets studied.

along the same feature dimensions as the smaller sets discussed in Sec. III A.

\section{CONCLUSION}

As was found by Chi et al., we agree that deep structure was an important feature determining the difference between experts and novices. For this reason it was important to construct a set of problems from a variety of chapters, our analog for "deep structure." Yet, the frequent null results obtained when replicating this experiment, as well as the results of our own statistical analysis, tell us that we must go beyond chapters and consider the pedagogical and cognitive properties of the problems that we select. We found that problems which ask students to perform different procedural tasks (e.g., making a flow chart of how you would solve a problem) are important to distinguish experts from novices. We also found that "easy" problems, as determined by a typical introductory physics textbook author, did a better job of discriminating experts from novices. This is not surprising: when a problem is so difficult that neither expert nor novice sorters can even figure out what the problem entails, it is likely going to be sorted randomly. Not surprisingly, larger problem sets still need to be carefully constructed along the feature dimensions we found.

While we agree that deep structure is an important feature determining the difference between experts and novices, we conclude that it is not the entire story. It is merely the largest pillar of a three pillar support which includes the procedural knowledge required to solve a problem as well as the difficulty of the problem as well. (See Fig. 3.) Not only is what you ask (deep structure) important, but also how you ask it. In order to differentiate

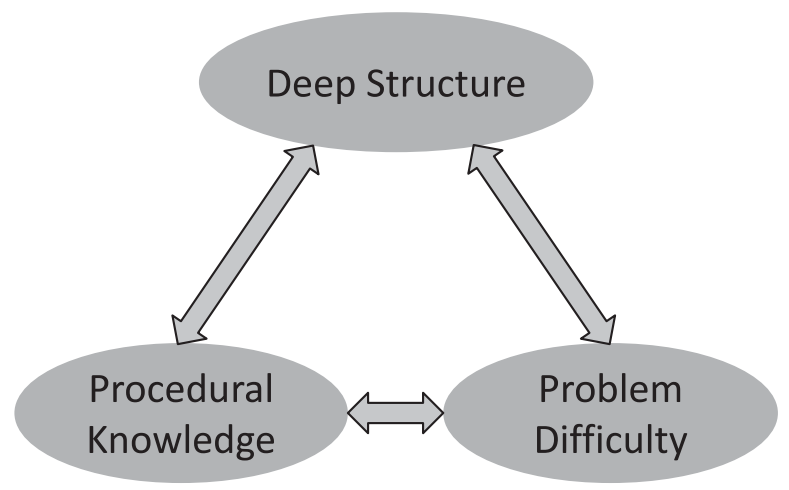

FIG. 3. The pillars of expert-novice differentiation.

experts from novices on a categorization task, we also need to ask questions which require different procedural tasks (e.g., make a flow chart). One might have hoped that these experiments would most strongly identify experts based on the types of declarative knowledge and procedural knowledge instead, which are arguably more authentic indicators of expertlikeness in real life. However, the relegation of deep structure from its former lonesome perch as the single salient feature which discriminates experts from novices to part of this triad is still a satisfying result which reflects the understanding gained since Chi et al. published their seminal result.

Even after considering all feature dimensions in this study, only $42.7 \%$ of the variability in the ten-problem set was explained. This means that there may be other latent variables, perhaps closely linked to the surface features in each problem, that need to be considered. Perhaps the remaining variability can only be resolved by intentionally planting surface features, as in Veldhuis' study [5]. Understanding this outstanding variability will be the key to predicting an instrument's ability to discriminate experts from novices.

\section{ACKNOWLEDGMENTS}

This work was supported by the CREATE for STEM Institute and Lyman Briggs College. The authors would like to thank the MSU physics faculty and the introductory physics classes in the Fall 2010-Spring 2011 semester for volunteering to sort problems for our study. One of the authors (S.F.W.) would also like to thank Raluca Teodorescu for her help in understanding the various nuances of the TIPP, as well as her assistance in constructing problems which would be diverse along both dimensions of the TIPP. Also, S.F.W. would like to thank Brian O'Shea for his help in getting an account on the High Performance Computer Cluster in order to run this analysis. One of the authors (G. K.) would also like to thank the group of David Pritchard at MIT for its hospitality during his sabbatical. 
[1] Michelene T. H. Chi, Paul J. Feltovich, and Robert Glaser, Categorization and representation of physics problems by experts and novices, Cogn. Sci. 5, 121 (1981).

[2] Steven F. Wolf, Daniel P. Dougherty, and Gerd Kortemeyer, Empirical approach to interpreting cardsorting data, Phys. Rev. ST Phys. Educ. Res. 8, 010124 (2012).

[3] Rachel E. Scherr, Modeling student thinking: An example from special relativity, Am. J. Phys. 75, 272 (2007).

[4] Brian W. Frank, Stephen E. Kanim, and Luanna S. Gomez, Accounting for variability in student responses to motion questions, Phys. Rev. ST Phys. Educ. Res. 4, 020102 (2008).

[5] G. Henry Veldhuis, The use of cluster analysis in categorization of physics problems, Sci. Educ. 74, 105 (1990).

[6] G. Henry Veldhuis, Differences in the categorization of physics problems by novices and experts, Ph.D. thesis, Iowa State University, 1986 (unpublished).

[7] Chandralekha Singh, Categorization of problems to assess and improve proficiency as teachers and learners, Am. J. Phys. 77, 73 (2009).

[8] James S. Walker, Physics (Pearson Education, Upper Saddle River, NJ, 2004), 2nd ed.

[9] Raluca Teodorescu, Cornelius Bennhold, and Gerald Feldman, Enhancing cognitive development through physics problem solving: A taxonomy of introductory physics problems, AIP Conf. Proc. 1064, 203 (2008).

[10] Harold Hotelling, The generalization of student's ratio, Ann. Math. Stat. 2, 360 (1931).

[11] L. Baringhaus and C. Franz, On a new multivariate twosample test, J. Multivariate Anal. 88, 190 (2004).

[12] B. A. Wiggins, Discriminant analysis of antibiotic resistance patterns in fecal streptococci, a method to differentiate human and animal sources of fecal pollution in natural waters, Appl. Environ. Microbiol. 62, 3997 (1996) [https://www.ncbi.nlm.nih.gov/pmc/articles/ PMC168217/].

[13] Harold Hotelling, Relations between two sets of variates, Biometrika 28, 321 (1936).

[14] Andrew Mason and Chandralekha Singh, Assessing expertise in introductory physics using categorization task, Phys. Rev. ST Phys. Educ. Res. 7, 020110 (2011).

[15] S. Kirkpatrick, C.D. Gelatt, Jr., and M.P. Vecchi, Optimization by simulated annealing, Science 220, 671 (1983).

[16] Claude J. P. Bélisle, Convergence theorems for a class of simulated annealing algorithms on $\mathbb{R}^{d}$, J. Appl. Probab. 29, 885 (1992). 